\title{
Advances in Optical and Magnetooptical Scatterometry of Periodically Ordered Nanostructured Arrays
}

\author{
Martin Veis and Roman Antos \\ Institute of Physics, Faculty of Mathematics and Physics, Charles University, Ke Karlovu 5, 12116 Prague, Czech Republic \\ Correspondence should be addressed to Martin Veis; veis@karlov.mff.cuni.cz
}

Received 26 November 2012; Accepted 27 December 2012

Academic Editor: Yue Li

Copyright (C) 2013 M. Veis and R. Antos. This is an open access article distributed under the Creative Commons Attribution License, which permits unrestricted use, distribution, and reproduction in any medium, provided the original work is properly cited.

\begin{abstract}
We review recent advances in optical and magnetooptical $(\mathrm{MO})$ scatterometry applied to periodically ordered nanostructures such as periodically patterned lines, wires, dots, or holes. The techniques are based on spectroscopic ellipsometry (SE), either in the basic or generalized modes, Mueller matrix polarimetry, and MO spectroscopy mainly based on MO Kerr effect measurements. We briefly present experimental setups, commonly used theoretical approaches, and experimental results obtained by SE and MO spectroscopic analyses of various samples. The reviewed analyses are mainly related to monitoring optical critical dimensions such as the widths, depths, and periods of the patterned elements, their real shapes, and their line edge or linewidth roughness. We also discuss the advantages and disadvantages of the optical spectroscopic techniques compared to direct monitoring techniques.
\end{abstract}

\section{Introduction}

Recent advances in nanotechnologies $[1,2]$ have yielded periodically ordered nanostructures with shapes of unlimited complexity, ultrahigh depth and lateral resolution, high aspect ratios, and various material compositions, all of which require further development of characterization techniques. Such techniques should measure the critical dimensions (CDs) of patterned lines, wires, dots, or holes with high accuracy and resolution, and also be capable of detecting tiny sidewall features of deep pattern trenches or other geometric parameters such as the line edge roughness (LER) or linewidth roughness (LWR) of patterned lines or wires.

The direct techniques for monitoring the geometric properties of laterally patterned nanostructures are scanning electron microscopy (SEM) and scanning probe methods such as scanning tunneling microscopy (STM), restricted to samples with metallic surfaces, atomic force microscopy (AFM), or magnetic force microscopy (MFM), restricted to samples with magnetic surfaces. Although these techniques have some disadvantages and restrictions (SEM requires cutting a sample to image its cross-section, while the scanning probe methods are affected by the probe-sample contact and special surfaces as mentioned), their recent development helped to overcome some of them. For instance, if an AFM probe uses a special tip with a flared apex radius [3] (so-called a CD tip) instead of a conventional tip, then the AFM measurement can yield an accurate relief profile, provided by an appropriate postprocessing algorithm which removes the tip's influence [4]. The direct techniques are also suitable for determining the LER/LWR parameters just by the statistical analysis of the scanned image $[5,6]$. However, the probe-sample contact still hinders the analysis and especially disables the LER measurement at the sharp bottom of patterned grooves.

The optical and magnetooptical (MO) spectroscopic techniques such as spectroscopic ellipsometry (SE) and magnetooptical Kerr effect (MOKE) measurements are not affected by the probe-sample contact. They are, however, indirect techniques that require both simulations and measurements of the optical spectra, from whose comparison, using a fitting procedure with the least square method, the requested parameters can be determined with the precision proportional to the amount of experimental data. The method is usually called optical or MO scatterometry.

In this paper we review some recent advances in the characterization of periodically patterned nanostructures by scatterometry based on SE and MOKE spectroscopy. We first introduce the experimental techniques used to measure SE 
and MO spectra, then we briefly list the most common theoretical approaches and their recent improvements, and finally we review some important results achieved on patterned nanostructures. For better demonstration, we also include an example of AFM, SE, and MO investigation of a magnetic grating.

\section{Experimental Techniques}

When polarization-sensitive measurements are employed to obtain experimental data, it is favourable to describe the polarization state of light by a proper mathematical approach. If depolarization effects are not considered, the Jones matrix formalism is frequently used for the description of polarized light and its transformation by polarizing optical elements. The optical and MO behavior of the sample can be macroscopically described within the Jones matrix formalism in the Cartesian representation (with the $s$ - and $p$-polarization bases), using the $2 \times 2$ Jones reflection matrix

$$
\mathbf{R}=\left[\begin{array}{ll}
r_{s s} & r_{s p} \\
r_{p s} & r_{p p}
\end{array}\right],
$$

where $r_{i j}$ are Fresnel amplitude reflection coefficients.

According to quantities measured, we can distinguish between photometric and ellipsometric experimental techniques. Spectrophotometry measures the amount of energy reflected or transmitted by the sample, so it provides the spectrally resolved information about $\left|r_{s s}\right|^{2}$ or $\left|r_{p p}\right|^{2}$. Typically, the light generated by a lamp is driven to the sample trough input optics and a polarizer. Then the reflected light intensity is detected by a photomultiplier or another type of photodetector. The main disadvantage of this technique is the time instability of the light flux and it also needs a proper calibration by reference reflectors.

Spectroscopic ellipsometry is an experimental technique that measures the change of incident light polarization upon its reflection (or sometimes transmission). The exact nature of polarization change is determined by the material and geometry of the nanostructured array. If the material of the sample is isotropic, then the Jones reflection matrix is diagonal and there is no interaction between the $s$ and $p$ waves. The diagonal elements are related to the array structure and its optical properties. The ellipsometric angles $\Psi$ and $\Delta$ are then defined by following equation:

$$
\varrho=\frac{r_{p p}}{r_{s s}}=\tan \Psi e^{j \Delta} .
$$

Spectral dependence of $\Psi$ and $\Delta$ obtained by SE carries the important information about the geometry of nanostructured arrays. Its analysis, however, requires advanced theoretical approaches, which are described in the next section. The principle of determining unknown parameters of an analyzed structure, called fitting, is minimizing the differences between simulated and measured values throughout the spectrum and all angles of incidence. More precisely, the error is here evaluated as the angular distances between measured and simulated point plotted on the Poincaré sphere, which is specified by the azimuthal angle $2 \Psi$ and the polar angle $\Delta$, that is, $\varepsilon_{j}=\mathbf{S}_{e, j} \cdot \mathbf{S}_{m, j}$, where the subscript " $e, j$ " means the $j$ th experimental value, " $m, j$ " means $j$ th modeled value, and the vectors $\mathbf{S}=[\sin 2 \Psi \cos \Delta, \sin 2 \Psi \sin \Delta, \cos 2 \Phi]$ denote the Stokes vectors of the experimental and modeled points on the sphere, respectively. For the sake of fitting, the sum of least squares $\varepsilon_{L S}^{2}=\sum_{j} \varepsilon_{j}^{2}$ is searched. However, finally the average error $\varepsilon=\left(\sum_{j=1}^{M} \varepsilon_{j}\right) / M$ is presented.

Typical ellipsometric experimental setup is the high power arc lamp-polarizer-sample-compensator-analyzer. This so-called PSCA optical sequence is widely used in standard ellipsometric setups. Detailed information about the main ideas of ellipsometry including basic notions on polarization and experimental techniques is treated in the book written by Azzam and Bashara [7]. Here, we restrict ourselves to the description of a specific four-zone ellipsometric configuration which was used in various scatterometric experiments [8-10]. This experiential setup is depicted in Figure 1. An optical sequence of this null ellipsometer is polarizer-sample-compensator-analyzer (PSCA), where the compensator is a phase-retarding tool. The Jones vector of the light at the output of the analyzer (and the intensity detected by the detector as well) depends on the rotation angles of polarizer, compensator, and analyzer: $\alpha$, $\beta, \gamma$. By searching the right values of $\alpha$ and $\beta$ at a fixed $\gamma$, the null ellipsometry provides a zero intensity of the light at the output. In the presented setup, the compensator is a quarterwave plate operating at two opposite positions $\gamma^{( \pm)}= \pm \pi / 2$. Taking this under consideration one has generally four null intensity positions of the optical setup. Therefore, it is favourable to divide the Poincaré sphere into four zones. From each zone, it is possible to derive the requested $\Psi$ and $\Delta$ quantities. To eliminate slight misalignments in the setup of polarizing components, the ellipsometric quantities are taken in all zones and finally their averages are calculated:

$$
\begin{aligned}
& \Psi=\frac{1}{4}\left(\overline{\alpha_{1}}-\overline{\alpha_{2}}+\overline{\alpha_{3}}-\overline{\alpha_{4}}\right), \\
& \Delta=\frac{1}{4}\left(\overline{\beta_{1}}+\overline{\beta_{2}}-\overline{\beta_{3}}-\overline{\beta_{4}}\right),
\end{aligned}
$$

where $\bar{\alpha}_{i}=\alpha_{i}-\pi / 2$ and $\overline{\beta_{i}}=\beta_{i}-\pi / 2$ are the null positions of the polarizer and analyzer corresponding to the $i$ th zone.

When an external magnetic field is applied to a ferromagnetic sample, it induces magnetooptical anisotropy. The off-diagonal elements of the Jones reflection matrix become in general nonzero, which indicates the conversion between $s$ incident and $p$ reflected $\left(r_{p s}\right)$ as well as $p$ incident and $s$ reflected $\left(r_{s p}\right)$ waves. Although the Jones reflection matrix has generally four elements, it can be simplified if the magnetic field is applied in the certain directions (e.g., perpendicular to the sample) [11].

There are two basic classes of experimental techniques which are employed for studies of weak MO effects. The first class is measuring the direct change of the intensity of light at the output of an optical element sequence. The second class, more sophisticated one, is based on the modulation of azimuth or ellipticity of incident light. In combination with a synchronic detection system, modulation techniques 


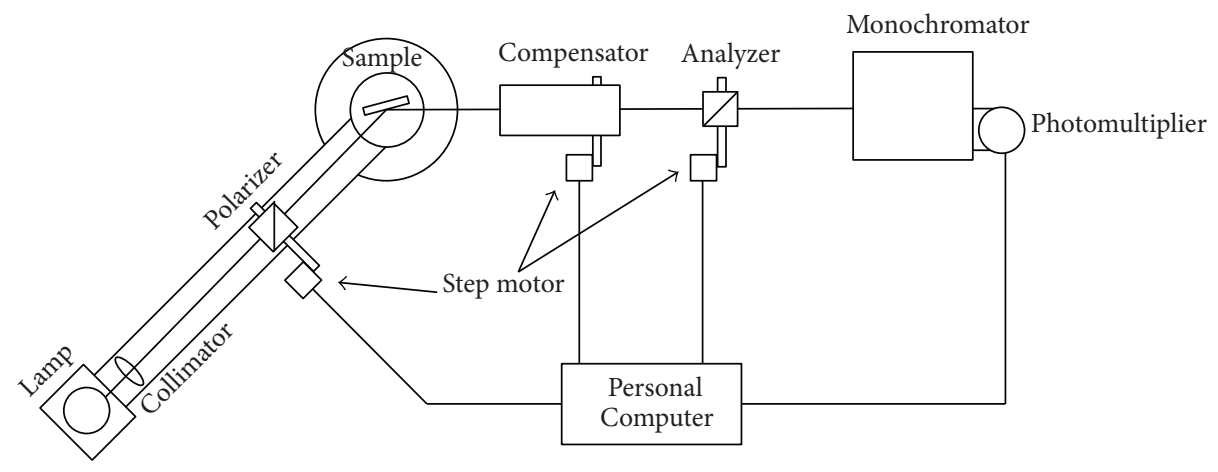

FIGURE 1: Experimental setup of four-zone null ellipsometer for scatterometric experiments.

increase the signal-to-noise ratio. However, both classes are used in different specific situations (Kerr microscopy, Kerr vector magnetometry, Kerr spectroscopy, etc.).

There are two basic techniques using the time modulation of the light polarization. First, an intensity-based method is based on the modulation of ellipticity of a light wave using the photoelastic modulator. This method allows fast measurement, which makes it suitable for studying dynamics of magnetization processes. Because it is an intensity method, the calibration of the equipment is required in each measurement, which makes it inconvenient for spectroscopic experiments. The second experimental technique employs the Faraday rotation in glass for the modulation of the azimuth of the polarization ellipse. Such a modulator, called the Faraday cell, consists of a fused quartz rod with optically polished faces, inserted into a solenoid coil. Owing to the sinusoidal time dependence of the modulation current in the coil generated by a high power audio amplifier, the azimuth of the light wave passing through the cell is harmonically modulated in time. One can imagine the Faraday cell as a rotator with time-dependent angle of rotation. A typical sequence of optical elements in a null MO spectrometer based on the azimuth modulation technique (depicted in Figure 2) is high power arc lamp-monochromator-polarizer- $d c$ compensating Faraday rotator-ac modulating Faraday rotator-phase plate (for Kerr ellipticity measurements)-sample-analyzerphotomultiplier. In the small angle approximation, the complex MOKE is measured as the ratio:

$$
\frac{r_{s p}}{r_{s s}} \approx \theta_{K}+i \epsilon_{K}
$$

or

$$
-\frac{r_{p s}}{r_{p p}} \approx \theta_{K}+i \epsilon_{K}
$$

where the indecent light beam is $s$ - or $p$-polarized, respectively. Here $\theta_{K}$ and $\epsilon_{K}$ denote the Kerr rotation and ellipticity. Compensating $d c$ Faraday cell automatically turns the plane of polarization by a certain angle to adjust detected intensity approximately to zero. Therefore, it compensates all changes produced by $\mathrm{MO}$ effects in the sample or by rotation of the polarizer. This Faraday cell is controlled by negative feedback loop. Measured signal on the output is then proportional to the magnetooptical effect of the sample and it decreases with increasing amplification of the feedback regulator. The negative feedback is introduced by means of analog output signal from the lock-in detector. This signal is used to control the current supply for the compensating cell.

Such compensation technique with azimuth modulation has several advantages over the other experimental methods. Azimuth modulation with synchronic detection notably increases the signal-to-noise ratio. The intensity dependence at the output is a linear function of the deviation from crossed polarizers. Crossing of the polarizers can be completely automatic (controlled by the negative feedback loop) and very precise without mechanical input. The value of the measured angle is not dependent on the intensity of output light (null method). Therefore if a highly stable current supply for the $d c$ compensating Faraday cell is used, very small MOKE angles down to the order of one millidegree can be measured. On the other hand, the usage of Faraday cells limits the photon energy range of the measured spectrum. Typically, the energy range is from 1.2 to $6 \mathrm{eV}$.

\section{Theoretical Approaches}

Periodic nanostructures or diffraction gratings can be classified by various means. According to the number of dimensions, they are one-, two-, or three-dimensional (1D, 2D, or 3D), where the third dimension generalizes a simple multilayer profile of the nanostructure within its depth. According to the pattern shape, there are sinusoidal and sinelike gratings (usually fabricated by holographic lithography) and lamellar gratings (usually made by exposure through masks combined with e-beam or ion-beam milling), and so forth. According to the used material, there are transparent and absorbing gratings, or isotropic and anisotropic gratings. The latter contain anisotropic materials, for example, magnetically ordered metals.

It is obvious that different modeling methods are suitable for different kinds of gratings. There is a variety of theoretical approaches, approximate and rigorous ones. Optics of shallow gratings, for example, can be advantageously simulated by an approximate theory based on local thin-film/multilayer approximation, neglecting the effect of patterned edges of 
nanoelements. This method, based on Fourier optics analysis of the sample illumination, is sometimes ambiguously referred to as the scalar diffraction theory. In this paper we call it the local modes method because it also works on anisotropic gratings requiring the fully vectorial electromagnetic approach [12]. Another type of nanostructures are subwavelength gratings, for example, wire-grid polarizers, which can be modeled by the effective medium theory where the periodic medium is regarded as an effective homogeneous medium.

General nanostructures, in particular structures with a larger period and deep trenches, require rigorous numerical methods based on electromagnetic theory. Among those, there are direct numerical approaches such as the finite difference method [13], finite elements method [14], or the finite difference time/frequency domain (FDTD/FDFD) method $[15,16]$, each of which can be used for any (a) periodic nanostructure. Then there are approaches originally used in other fields or applications, for example, the plane wave expansion method, Korringa-Kohn-Rostoker [17] and other methods based on Green's functions, method of fictitious sources [18], variational methods [19], or diffractive approaches. Finally, there are methods originally developed for diffraction gratings $[20,21]$, which can be classified as differential, integral, and modal methods (according to the chosen numerical algorithm). They are based on the Fourier expansion of the electromagnetic fields and either the material parameters (the coupled-wave method and Fourier modal method) or the relief functions (Chandezon's coordinate transformation method [22], appropriate for sinusoidal gratings).

The coupled-wave method, usually called the rigorous coupled-wave analysis (RCWA) [23], is one of the most used approaches to simulate the optical response of general periodic nanostructures and is also used in simulations presented in this paper. During the latter half of the last century, it underwent a remarkable progress towards high accuracy and generality. Since Kogelnik's paper [24], which used only two waves in a volume grating, the method was generalized and improved to simulate with high-precision deep, 2D, arbitrary-relief, and anisotropic gratings and their various combinations. The improvements have been achieved by improving propagational algorithms, from the transfermatrix algorithm, through the R-matrix algorithm, to the presently most used scattering-matrix algorithm [25]. Further enhancements of the methods have been performed by modifying the Fourier factorization procedures according to the field discontinuities at the pattern boundaries [26-30], and other modern enhancements such as the adaptive spatial resolution (ASR) [31].

\section{Results Obtained by Scatterometry}

4.1. Purely Optical Scatterometry. Optical scatterometry is most frequently based on SE and sometimes is combined with spectrophotometry. The SE spectra are almost always measured in the specular reflection, which corresponds to the zeroth order of the grating diffraction. In the case of $1 \mathrm{D}$ gratings, the plane of incidence is usually chosen perpendicular to the grating grooves, which corresponds to planar diffraction (the entire problem is then solved in one plane for each independent polarization, which reduces the computation time). Using the specular SE, various authors have successfully determined linewidths, periods, depths, and other fine profile features, as already reviewed by Huang and Terry [32]. It has been shown that some of the features detected by scatterometry are not detectable by scanning probe methods (e.g., sharp bottom corners of grooves) and are difficult to observe on SEM images.

Obvious advantages of scatterometry (besides it is nondestructive) are higher sensitivity, no contact with any mechanical tool, and possibility to monitor features beneath the relief such as native oxide overlayers or thermally induced overlayers developed in an oxygen/nitrogen atmosphere. Recently, Ghong et al. [33] have demonstrated the influence of the native oxide overlayer on the SE spectra measured on $1 \mathrm{D}$ and 2D silicon gratings. Similarly, Yu et al. [34] used SE to monitor a coating process when a resist layer was deposited over a silicon grating.

On the other hand, scatterometry has some disadvantages: spectral measurements are indirect, and the measured spectra sometimes require very difficult analyses to reveal the real profile of the structure, which should be approximately known before starting the fitting procedure (to use it as an initial value). Moreover, the spectra can contain too many unknown parameters, or at least some vaguely known parameters. For these reasons, it is important to study carefully the influence of the geometric and material parameters on the spectral dependences of the optical response $[35,36]$. Furthermore, Kim et al. [37] have shown that the detailed theoretical analysis of the SE dependence on the geometric parameters can also help one to determine asymmetric profiles of gratings, although the SE response is same when light illuminates the asymmetric grooves from either the left or the right side.

Another disadvantage of scatterometry is the long computation time during the fitting procedure when complicated structures are analyzed. Therefore, for computation it is desirable to retain as few Fourier components of the periodic functions as possible but to still have good approximation [8].

To overcome the problem of many parameters and to increase the sensitivity of SE measurements, novel ellipsometric configurations have been proposed. For instance, Liu and Terry Jr. [38] have developed immersion scatterometry which used SE in an immersion mode (in water), which increased the refractive index of the grating's ambient medium and thus increased the numerical aperture of the optical apparatus.

Other configurations that increase the sensitivity and the number of detectable parameters are generalized ellipsometry [39, 40] and Mueller matrix polarimetry [32]. Both methods are capable to deal with anisotropic samples, where anisotropy might exist either due to the presence of anisotropic materials (such as ordered uniaxial or biaxial nanocrystals) or due to the order of nanoelements, where both cases yield small cross-polarization effects not detectable by the conventional SE. Using both methods, 
Foldyna et al. [41, 42] have accurately determined the depth and the top width of 2D-periodic square holes patterned in the photoresist film deposited on a silicon substrate and discussed possibilities of determining further parameters from the same spectra.

Unlike generalized ellipsometry, the Mueller matrix polarimetry works not only with cross-polarization effects, but also can detect the depolarization effects caused by surface roughness or LER and LWR. Foldyna et al. [43] demonstrated the application of this method to monitor the LWR.

As an example of scatterometric analysis based on SE measurement, consider a rectangular-relief grating patterned on the top of a $32 \mathrm{~nm}$ thick permalloy (NiFe) film deposited on a $\mathrm{Si}$ substrate, whose AFM measurement is displayed in Figure 3 (the details of fabrication are described in [44]). The comparison of the nominal geometric parameters (those intended by the grating manufacturer) with parameters determined by AFM and scatterometry (based on SE performed at three angles of incidence, 60, 70, and $80^{\circ}$, the last of which is displayed in Figure 4, using Permalloy's material parameters from [45]) is listed in Table 1.

Here the AFM measurement (in the conventional tip mode) provides a direct image of the grating's relief profile, but the horizontal values (period and linewidth) are affected by a $9 \%$ error due to wrong scale. According to our experiences, the period of patterns made by lithographic processes is always achieved with high precision, so that we scale the horizontal AFM parameters to obtain the nominal $1000 \mathrm{~nm}$ period and to keep the same period-to-linewidth ratio (the vertical depth $21.7 \mathrm{~nm}$ is kept without change). The scaled AFM linewidth $(329.3 \mathrm{~nm}$, measured at the bottom) now corresponds well to the linewidth determined by scatterometry $(307.2 \mathrm{~nm})$; the $22 \mathrm{~nm}$ difference is probably due to the finite size of the apex of the AFM tip. Since the presented ellipsometric spectrum is not sensitive to the grating period (due to the shallow relief), it was not fitted but just verified by the spectral positions of Wood anomalies (the tiny discontinuities in the spectra).

Finally, the $2.6 \mathrm{~nm}$ difference between the AFM and scatterometric values of the relief depth is most probably due to some negligence of the used optical model such as the native top $\mathrm{NiFe}$ oxide overlayer or inaccurate $\mathrm{NiFe}$ optical parameters. This is also the reason why it is difficult to determine the real error of the fitted parameters (or the possible range of their accurate values). Instead, the error of the fitted optical parameters $(\Psi$ and $\Delta)$ is usually listed (determined as the average difference on the Poincaré sphere in degrees), which approximately reflects the quality of the fit. This error is in our case $\varepsilon=2.8^{\circ}$.

4.2. Magnetooptical Scatterometry. Another way how to increase accuracy or the number of detectable parameters is measuring higher diffraction orders or additional spectra such as MO spectroscopy, which can be also regarded as a generalized SE method applicable to magnetic gratings.

Laterally patterned magnetic nanostructures [46] were originally investigated with respect to their micromagnetic
TABLE 1: Comparison of grating geometrical parameters obtained by AFM and scatterometry, together with nominal ones (intended by the manufacturer). All the dimensions are in nanometers.

\begin{tabular}{|c|c|c|c|c|}
\hline Parameter & Nominal & AFM & Scaled AFM & Scatterometry \\
\hline Period & 1000 & 1091.5 & $1000^{\star}$ & $1000^{\star \star}$ \\
\hline Linewidth & 500 & 359.4 & 329.3 & $307.2^{\star \star \star}$ \\
\hline NiFe thickness & 32 & - & - & - \\
\hline Relief depth & 16 & 21.7 & 21.7 & $24.3^{\star \star \star}$ \\
\hline
\end{tabular}

${ }^{\star}$ Fixed value.

${ }^{\star *}$ Just verified from the position of the Wood anomaly.

${ }^{\star \star \star}$ The average error of the fit on Poincaré sphere is $\varepsilon=3.8^{\circ}$.

properties, that is, the switching behavior [47] and later also spin-dynamical processes [48]. Besides MFM [49] and spin-polarized STM [50], MO experiments were also employed. Single-wavelength MOKE measurements in specular reflectin and higher diffraction orders became the basis for the technique called diffracted MOKE (D-MOKE) [47, 51], which provides an approximate geometrical distribution of magnetization within a periodic magnetic nanostructure. In the D-MOKE measurement, this information is distributed among the beams diffracted by the nanostructure's periodicity (where higher diffraction orders are more sensitive to the vicinity of the edges of magnetized elements).

A more precise magnetization distribution can be measured by microscopic MOKE (M-MOKE), which does not require the periodic arrangement of magnetic elements. In the wide-field mode [52], the direct magnified image of a single element or a tiny array of elements is provided being sensitive to one chosen magnetization component. In the scanning mode [48], the surface is scanned while the magnetization vector is determined at each point by analyzing asymmetries in the optical Fourier transform image.

The essential advantage of both $\mathrm{D}$ - and M-MOKE measurements and the reason for their popularity is again the simplicity of the data analysis, providing the direct or nearly direct magnetic image of the illuminated sample. However, these methods are affected by limitations of the optical imaging and by imperfections of the investigated patterns. For instance, the periodicity of an analyzed structure comparable to the wavelength of detection light makes it almost impossible to record the sufficient number of higher diffraction orders for a proper D-MOKE analysis. Next, the M-MOKE is limited by the detection resolution, also affected by the nonzero wavelength and by the numerical aperture of the objective lens. And finally, the M-MOKE wide-field and scanned images are strongly affected by the so-called edge effects of the lateral patterning, that is, the effects of internal diffraction by the edges of the patterned elements. The edge effects blur the recorded image and thus erase the information about magnetization near the vicinity of the magnetized elements. Particularly important edge effects affecting the MOKE approaches are the cross-polarization effects, observed when the angle of the incidence plane is oblique to the edges, and the depolarization effects, mainly caused by the LER. 


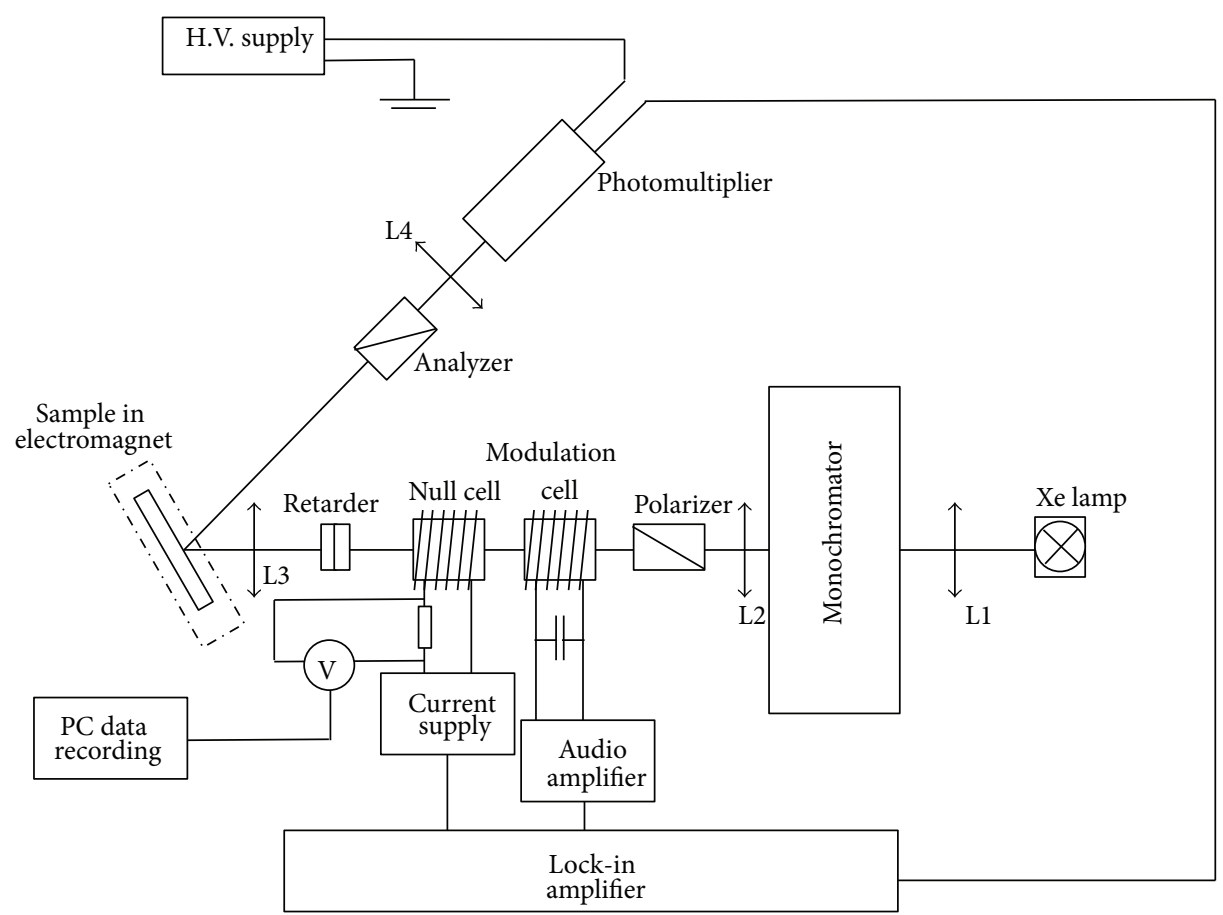

Figure 2: Experimental setup for MOKE measurements utilizing azimuth modulation in the photon energy range from 1.2 to $6 \mathrm{eV}$. L1, L2, L3, and L4 denote quartz lenses.

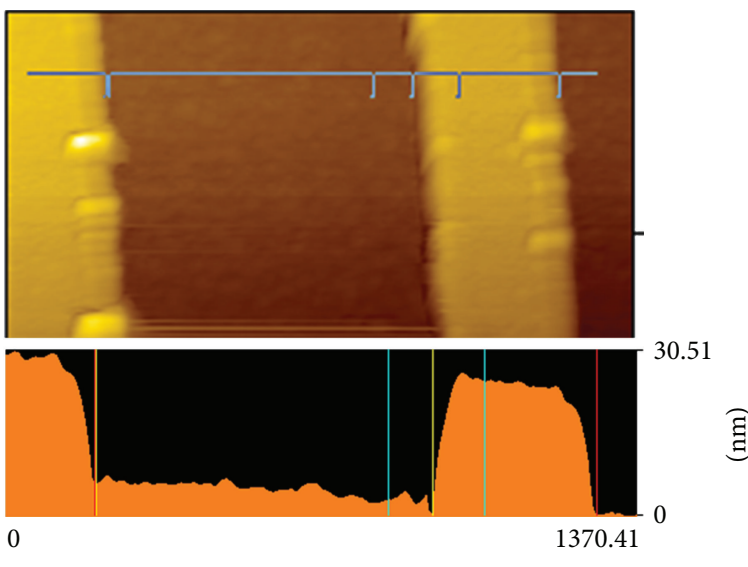

$(\mathrm{nm})$

FIGURE 3: AFM measurement on a NiFe grating.

The aim to use the MOKE techniques even beyond those limitations demands to investigate those effects by a method which would accurately distinguish between the magnetization response and the effects due to optical imaging and fabrication imperfections. Several papers $[9,10$, $12,44]$ have already shown that this demand is satisfied by MO spectroscopic scatterometry, which can be applied as accurate quality control of lithographic manufacturing or as a tool quantitatively determining the LER, native oxide, and other effects, so that it could be later utilized for extending the D- and M-MOKE analyses to higherresolution patterns. It is worth pointing out that MO spectroscopic analyses could take place after the development of proper modeling methods for anisotropic gratings, which are considerably more difficult than those for isotropic ones [53-56].

In particular, [9] demonstrates that MOKE spectra measured in zeroth and -1 st diffraction orders can be used to determine the thicknesses of native oxide overlayers developed on the top of a magnetic grating (made here as periodic NiFe wires capped by a protection $\mathrm{Cr}$ overlayer, fabricated on the top of a Si substrate). It was shown that the zeroth order was particularly sensitive to the $\mathrm{SiO}_{2}$ overlayer developed on the top of the Si substrate before fabricating the grating. On the other hand, the -1 st order was more sensitive to the $\mathrm{Cr}_{2} \mathrm{O}_{3}$ overlayer developed on the top of the Cr capping.

It was further shown [10] that applying $p$-polarized incident light in the MOKE measurement helps one to determine the quality of the edges of the patterned wires by a numerical quantity denoted $\eta$, which is proportional to the smoothness of the edges $(\eta=1$ means that the edges are perfect, whereas $\eta=0$ means that the LER and LWR are so large that the patterned area is randomly disordered). Measurements of $\eta$ on two examples of gratings displayed in Figure 5 yielded the values $\eta=7.0$ (Figure 5(a)) and $\eta=0.53$ (Figure 5(b)), which clearly corresponds with the obvious quality of the samples. The parameter $\eta$ can be, therefore, identified with the grating's LER. 


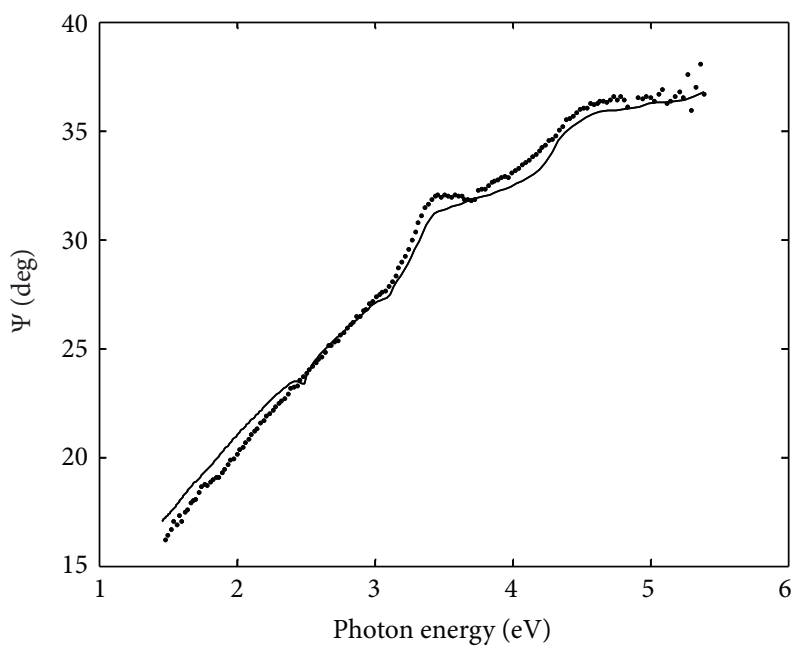

(a)

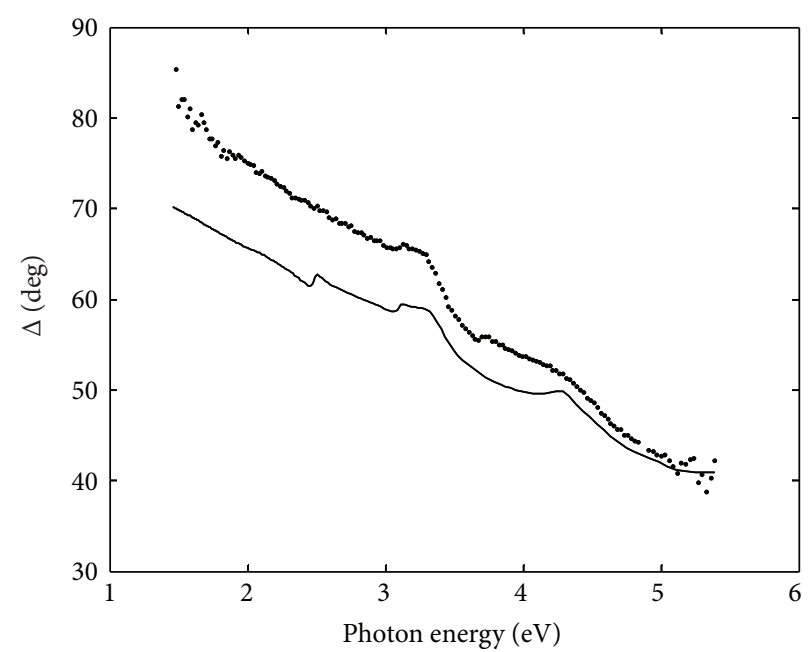

(b)

FIGURE 4: Spectroellipsometric parameters $\Psi$ and $\Delta$ measured (dots) and simulated (solid curves) on the NiFe rectangular-relief grating with the angle of incidence of $80^{\circ}$. The geometric parameters for the simulation were chosen those in the last column of Table 1.
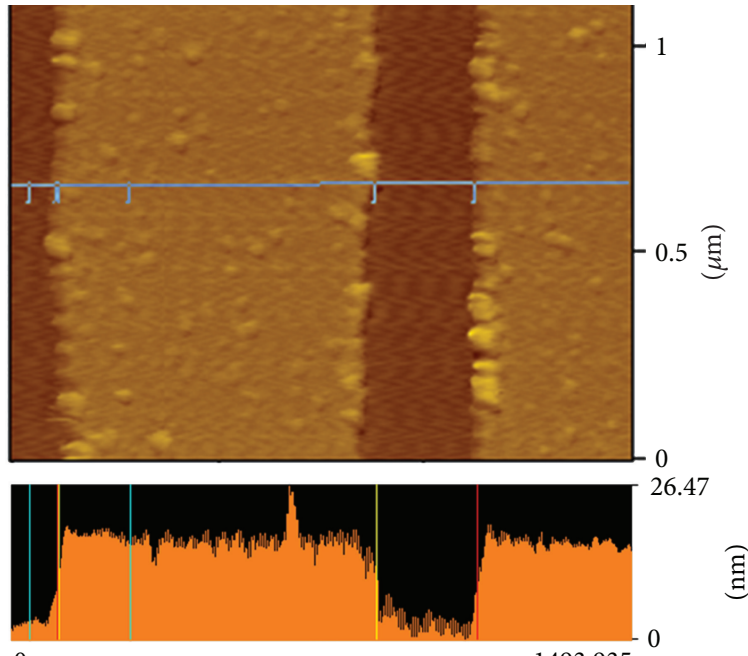

$(\mathrm{nm})$
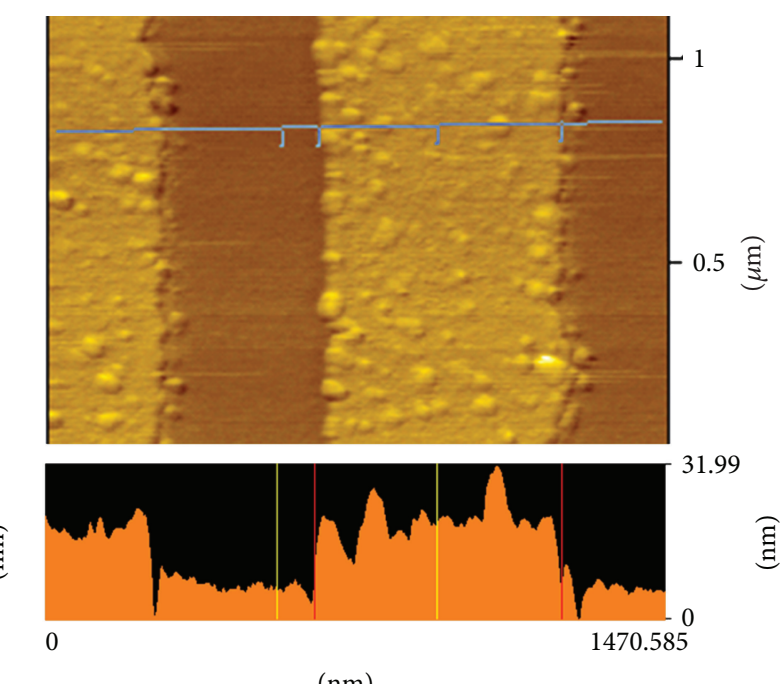

$(\mathrm{nm})$

(a)

(b)

FIgURE 5: AFM measurements of two $\mathrm{Cr} / \mathrm{NiFe}$ wire samples made on a Si substrate, both with different LER.

To illustrate difficulties coupled with too high sensitivity of the MO spectroscopy, Figure 6 shows a comparison of measured and simulated MOKE parameters, the Kerr rotation and ellipticity in the polar magnetic configuration, using again the material parameters from [45] and the grating's geometrical dimensions determined by SE. The discrepancy between the theory end experiment in some spectral ranges are again due to possible inaccuracy of the material parameters and/or the native NiFe oxide overlayer.

\section{Conclusions}

We have reviewed some recent advances in optical and MO characterization techniques (mainly based on SE and MOKE spectroscopy) applied to various grating structures and discussed their advantages and disadvantages as compared to direct monitoring techniques such as SEM, AFM, STM (spin polarized STM for magnetic samples), MFM, DMOKE, and M-MOKE. The spectroscopic techniques require 


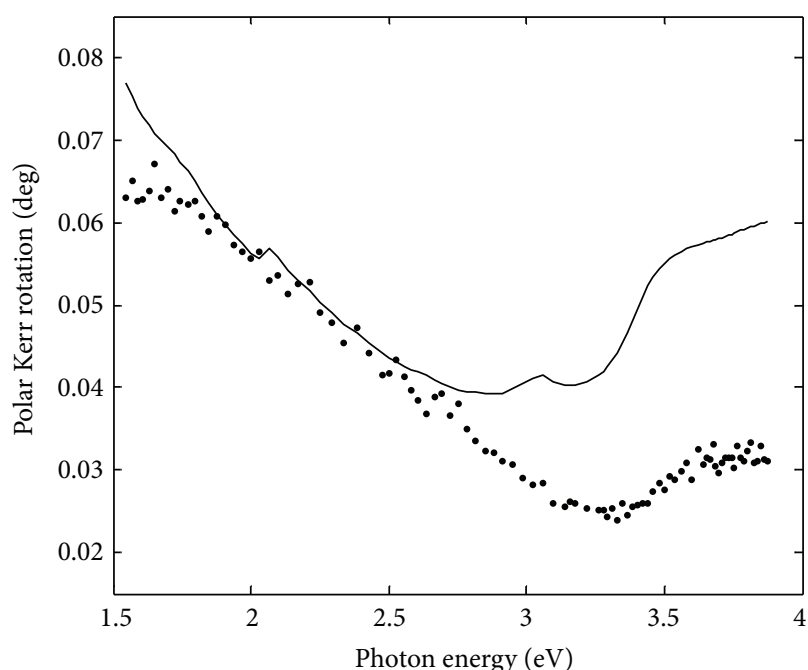

(a)

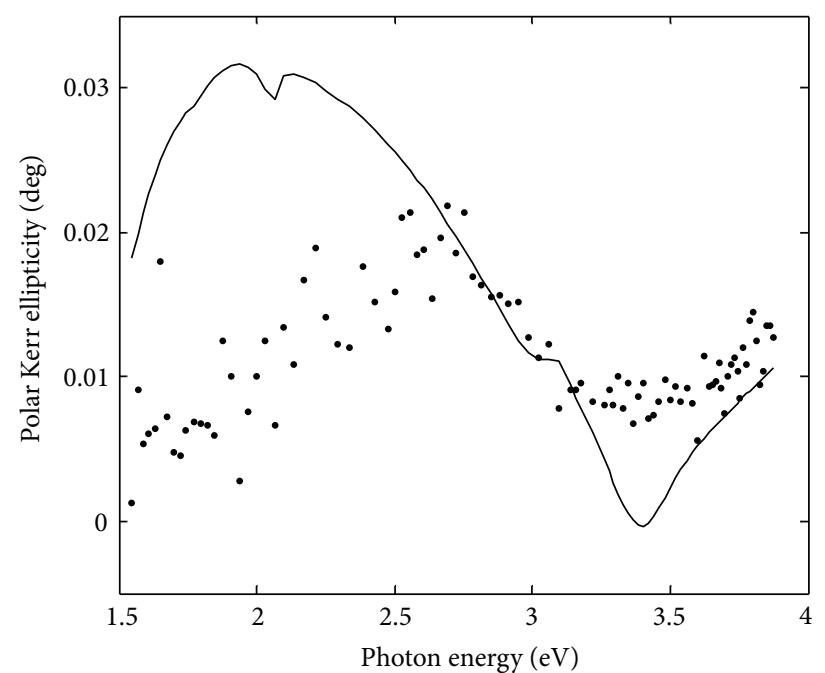

(b)

FIGURE 6: MOKE parameters (polar Kerr rotation and ellipticity) measured (dots) and simulated (solid curves) on the permalloy rectangularrelief grating, the same as presented in Figures 3 and 4.

more difficult and time-consuming postprocessing analysis of measured experimental data. However, they are more sensitive, nondestructive, and capable of detecting more unknown parameters. Besides monitoring optical CDs, they can also evaluate such parameters like LER or LWR.

\section{Acknowledgment}

The work was supported by Czech Science Foundation Grant no. P204/10/P346.

\section{References}

[1] C. Lu and R. H. Lipson, "Interference lithography: a powerful tool for fabricating periodic structures," Laser \& Photonics Reviews, vol. 4, no. 4, pp. 568-580, 2010.

[2] S. Brose, S. Danylyuk, L. Juschkin et al., "Broadband transmission masks, gratings and filters for extreme ultraviolet and soft X-ray lithography," Thin Solid Films, vol. 520, no. 15, pp. 5080-5085, 2012.

[3] H. Liu, M. Klonowski, D. Kneeburg, G. Dahlen, M. Osborn, and T. Bao, "Advanced atomic force microscopy probes: wear resistant designs," Journal of Vacuum Science and Technology B, vol. 23, no. 6, pp. 3090-3093, 2005.

[4] G. Dahlen, M. Osborn, N. Okulan, W. Foreman, A. Chand, and J. Foucher, "Tip characterization and surface reconstruction of complex structures with critical dimension atomic force microscopy," Journal of Vacuum Science and Technology B, vol. 23, no. 6, pp. 2297-2303, 2005.

[5] J. Thiault, J. Foucher, J. H. Tortai, O. Joubert, S. Landis, and S. Pauliac, "Line edge roughness characterization with a three-dimensional atomic force microscope: transfer during gate patterning processes," Journal of Vacuum Science and Technology B, vol. 23, no. 6, pp. 3075-3079, 2005.

[6] Y. Ma and F. Cerrina, "Effect of a surface inhibition layer on line edge roughness," Journal of Vacuum Science and Technology B, vol. 23, no. 3, pp. 1096-1101, 2005.
[7] R. A. M. Azzam and N. M. Bashara, Ellipsometry and Polarized Light, North Holland Publishing, North-Holland, The Netherlands, 1977.

[8] R. Antos, J. Pistora, J. Mistrik et al., "Convergence properties of critical dimension measurements by spectroscopic ellipsometry on gratings made of various materials," Journal of Applied Physics, vol. 100, no. 5, Article ID 054906, 2006.

[9] R. Antos, J. Mistrik, T. Yamaguchi, S. Visnovsky, S. O. Demokritov, and B. Hillebrands, "Evidence of native oxides on the capping and substrate of permalloy gratings by magneto-optical spectroscopy in the zeroth- and first-diffraction orders," Applied Physics Letters, vol. 86, no. 23, Article ID 231101, 3 pages, 2005.

[10] R. Antos, J. Mistrik, T. Yamaguchi, S. Visnovsky, S. O. Demokritov, and B. Hillebrands, "Evaluation of the quality of permalloy gratings by diffracted magneto-optical spectroscopy," Optics Express, vol. 13, no. 12, pp. 4651-4656, 2005.

[11] S. Visnovsky, Optics in Magnetic Multilayers and Nanostructures, CRC Press, Taylor \& Francis, Boca Raton, Fla, USA, 2006.

[12] R. Antoš, J. Mistrík, M. Aoyama, T. Yamaguchi, S. Visnovsky, and B. Hillebrands, "Magneto-optical spectroscopy on permalloy wires in 0th and 1st diffraction orders," Journal of Magnetism and Magnetic Materials, vol. 272, part 3, supplement 1, pp. 1670-1671, 2004.

[13] P. Vincent, "A finite-difference method for dielectric and conducting crossed gratings," Optics Communications, vol. 26, no. 3, pp. 293-296, 1978.

[14] T. Delort and D. Maystre, "Finite-element method for gratings," Journal of the Optical Society of America A, vol. 10, no. 12, pp. 2592-2601, 1993.

[15] H. Ichikawa, "Electromagnetic analysis of diffraction gratings by the finite-difference time-domain method," Journal of the Optical Society of America A, vol. 15, no. 1, pp. 152-157, 1998.

[16] S. D. Wu and E. N. Glytsis, "Volume holographic grating couplers: rigorous analysis by use of the finite-difference frequencydomain method," Applied Optics, vol. 43, no. 5, pp. 1009-1023, 2004. 
[17] A. Modinos, N. Stefanou, and V. Yannopapas, "Applications of the layer-KKR method to photonic crystals," Optics Express, vol. 8, no. 3, pp. 197-202, 2001.

[18] F. Zolla and R. Petit, "Method of fictitious sources as applied to the electromagnetic diffraction of a plane wave by a grating in conical diffraction mounts," Journal of the Optical Society of America A, vol. 13, no. 4, pp. 796-802, 1996.

[19] O. P. Bruno and F. Reitich, "Numerical solution of diffraction problems: a method of variation of boundaries," Journal of the Optical Society of America A, vol. 10, no. 6, pp. 1168-1175, 1993.

[20] R. Petit, Ed., Electromagnetic Theory of Gratings, Springer, Berlin, Germany, 1980.

[21] E. G. Loewen and E. Popov, Diffraction Gratings and Applications, CRC Press, New York, NY, USA, 1997.

[22] L. Li and J. Chandezon, "Improvement of the coordinate transformation method for surface-relief gratings with sharp edges," Journal of the Optical Society of America A, vol. 13, no. 11, pp. 2247-2255, 1996.

[23] M. G. Moharam and T. K. Gaylord, "Rigorous coupled-wave analysis of planar-grating diffraction," Journal of the Optical Society of America, vol. 71, no. 7, pp. 811-818, 1981.

[24] H. Kogelnik, "Coupled wave theory for thick hologram gratings," The Bell System Technical Journal, vol. 48, no. 9, pp. 2909-2947, 1969.

[25] N. P. K. Cotter, T. W. Preist, and J. R. Sambles, "Scattering-matrix approach to multilayer diffraction," Journal of the Optical Society of America A, vol. 12, no. 5, pp. 1097-1103, 1995.

[26] P. Lalanne and G. M. Morris, "Highly improved convergence of the coupled-wave method for TM polarization," Journal of the Optical Society of America A, vol. 13, no. 4, pp. 779-784, 1996.

[27] P. Lalanne, "Convergence performance of the coupled-wave and the differential methods for thin gratings," Journal of the Optical Society of America A, vol. 14, no. 7, pp. 1583-1591, 1997.

[28] P. Lalanne, "Improved formulation of the coupled-wave method for two-dimensional gratings," Journal of the Optical Society of America A, vol. 14, no. 7, pp. 1592-1598, 1997.

[29] L. Li, "Use of Fourier series in the analysis of discontinuous periodic structures," Journal of the Optical Society of America A, vol. 13, no. 9, pp. 1870-1876, 1996.

[30] R. Antos, "Fourier factorization with complex polarization bases in modeling optics of discontinuous bi-periodic structures," Optics Express, vol. 17, no. 9, pp. 7269-7274, 2009.

[31] G. Granet, "Reformulation of the lamellar grating problem through the concept of adaptive spatial resolution," Journal of the Optical Society of America A, vol. 16, no. 10, pp. 2510-2516, 1999.

[32] H. T. Huang and F. L. Terry Jr., "Spectroscopic ellipsometry and reflectometry from gratings (Scatterometry) for critical dimension measurement and in situ, real-time process monitoring," Thin Solid Films, vol. 455-456, no. 1-2, pp. 828-836, 2004.

[33] T. H. Ghong, J. S. Byun, S. H. Han, J. M. Chung, and Y. D. Kim, "Analysis of 2D periodic nanostructures with an oxide overlayer via spectroscopic ellipsometry," Journal of Nanoscience and Nanotechnology, vol. 11, supplement I, no. 7, pp. 6514-6517, 2011.

[34] Z. Yu, J. Hwu, Y. Liu, G. Gauzner, K. Lee, and D. Kuo, "Study of spin-coated resist coverage on nanoscale topography using spectroscopic ellipsometry," Journal of Applied Physics, vol. 110, no. 1, Article ID 014303, 2011.

[35] R. Antos, I. Ohlidal, J. Mistrik et al., "Spectroscopic ellipsometry on lamellar gratings," Applied Surface Science, vol. 244, no. 1-4, pp. 225-229, 2005.
[36] R. Antos, J. Pistora, I. Ohlidal et al., "Specular spectroscopic ellipsometry for the critical dimension monitoring of gratings fabricated on a thick transparent plate," Journal of Applied Physics, vol. 97, no. 5, Article ID 053107, 7 pages, 2005.

[37] T. J. Kim, S. H. Han, T. H. Ghong, J. S. Byun, J. M. Chung, and Y. D. Kim, "Study on an asymmetric nanostructure by using a rigorous coupled-wave analysis," Journal of the Korean Physical Society, vol. 56, no. 41, pp. 1278-1281, 2010.

[38] E. Liu and F. L. Terry Jr., "Immersion scatterometry for improved nano-scale topography measurements," Physica Status Solidi A, vol. 205, no. 4, pp. 784-788, 2008.

[39] M. Schubert, "Generalized ellipsometry and complex optical systems," Thin Solid Films, vol. 313-314, pp. 323-332, 1998.

[40] L. Halagačka, K. Postava, M. Foldyna, and J. Pištora, "Precise phase-modulation generalized ellipsometry of anisotropic samples," Physica Status Solidi A, vol. 205, no. 4, pp. 752-755, 2008.

[41] M. Foldyna, A. De Martino, E. Garcia-Caurel et al., "Monitoring critical dimensions of bidimensional gratings by spectroscopic ellipsometry and mueller polarimetry," Physica Status Solidi A, vol. 205, no. 4, pp. 806-809, 2008.

[42] M. Foldyna, A. De Martino, E. Garcia-Caurel et al., "Critical dimension of biperiodic gratings determined by spectral ellipsometry and Mueller matrix polarimetry," The European Physical Journal of Applied Physics, vol. 42, no. 3, pp. 351-359, 2008.

[43] M. Foldyna, T. A. Germer, B. C. Bergner, and R. G. Dixson, "Generalized ellipsometry of artificially designed line width roughness," Thin Solid Films, vol. 519, supplement 1, no. 9, pp. 2633-2636, 2011.

[44] R. Antos, M. Veis, E. Liskova et al., "Optical metrology of patterned magnetic structures: deep versus shallow gratings," in Metrology, Inspection, and Process Control for Microlithography XIX, vol. 5752 of Proceedings of SPIE, pp. 1050-1059, San Jose, Calif, USA, March 2005.

[45] G. Neuber, R. Rauer, J. Kunze et al., “Temperature-dependent spectral generalized magneto-optical ellipsometry," Applied Physics Letters, vol. 83, no. 22, pp. 4509-4511, 2003.

[46] J. I. Martin, J. Nogues, K. Liu, J. L. Vicent, and I. K. Schuller, "Ordered magnetic nanostructures: fabrication and properties," Journal of Magnetism and Magnetic Materials, vol. 256, no. 1-3, pp. 449-501, 2003.

[47] M. Grimsditch and P. Vavassori, "The diffracted magneto-optic Kerr effect: what does it tell you?" Journal of Physics Condensed Matter, vol. 16, no. 9, pp. R275-R294, 2004.

[48] W. K. Hiebert, A. Stankiewicz, and M. R. Freeman, "Direct observation of magnetic relaxation in a small permalloy disk by time-resolved scanning Kerr microscopy," Physical Review Letters, vol. 79, no. 6, pp. 1134-1137, 1997.

[49] T. Shinjo, T. Okuno, R. Hassdorf, K. Shigeto, and T. Ono, "Magnetic vortex core observation in circular dots of permalloy," Science, vol. 289, no. 5481, pp. 930-932, 2000.

[50] A. Wachowiak, J. Wiebe, M. Bode, O. Pietzsch, M. Morgenstern, and R. Wiesendanger, "Direct observation of internal spin structure of magnetic vortex cores," Science, vol. 298, no. 5593, pp. 577-580, 2002.

[51] J. B. Kim, Y. H. Lu, M. H. Cho et al., "Diffracted magneto-optical Kerr effect of a Ni magnetic grating," Journal of Applied Physics, vol. 106, no. 9, Article ID 093103, 2009.

[52] A. Neudert, J. McCord, R. Schäfer, and L. Schultz, "Subnanosecond vortex transformation in ferromagnetic film elements observed by stroboscopic wide-field Kerr microscopy," Journal 
of Applied Physics, vol. 97, no. 10, part 2, Article ID 10E701, 3 pages, 2005.

[53] K. Rokushima and J. Yamakita, "Analysis of anisotropic dielectric gratings," Journal of the Optical Society of America, vol. 73, no. 7, pp. 901-908, 1983.

[54] S. Visnovsky and K. Yasumoto, "Multilayer anisotropic biperiodic diffraction gratings," Czechoslovak Journal of Physics, vol. 51, no. 3, pp. 229-247, 2001.

[55] M. H. Cho, Y. Lu, J. Y. Rhee, and Y. P. Lee, "Rigorous approach on diffracted magneto-optical effects from polar and longitudinal gyrotropic gratings," Optics Express, vol. 16, no. 21, pp. 16825-16839, 2008.

[56] M. H. Cho, H. Zheng, Y. Lu, Y. Lee, and W. Cai, "Improved rigorous coupled-wave analysis for polar magnetic gratings," Computer Physics Communications, vol. 182, no. 2, pp. 360-365, 2011. 

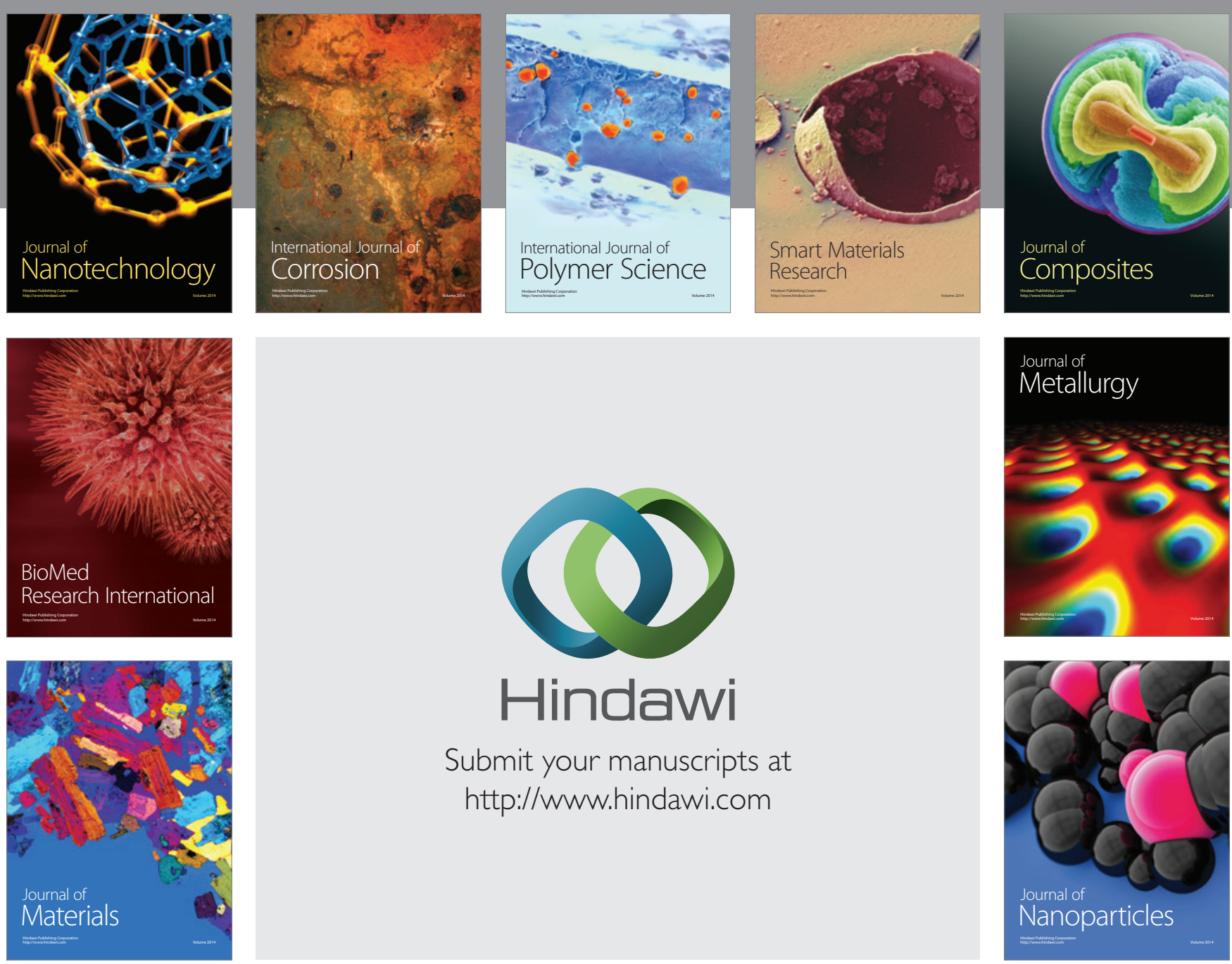

Submit your manuscripts at http://www.hindawi.com
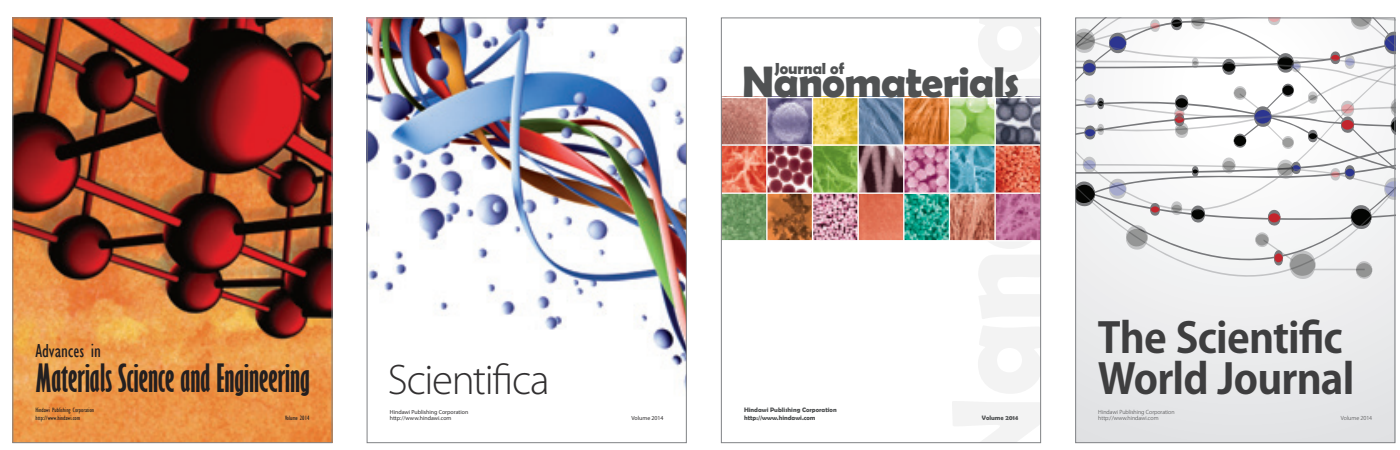

\section{The Scientific World Journal}
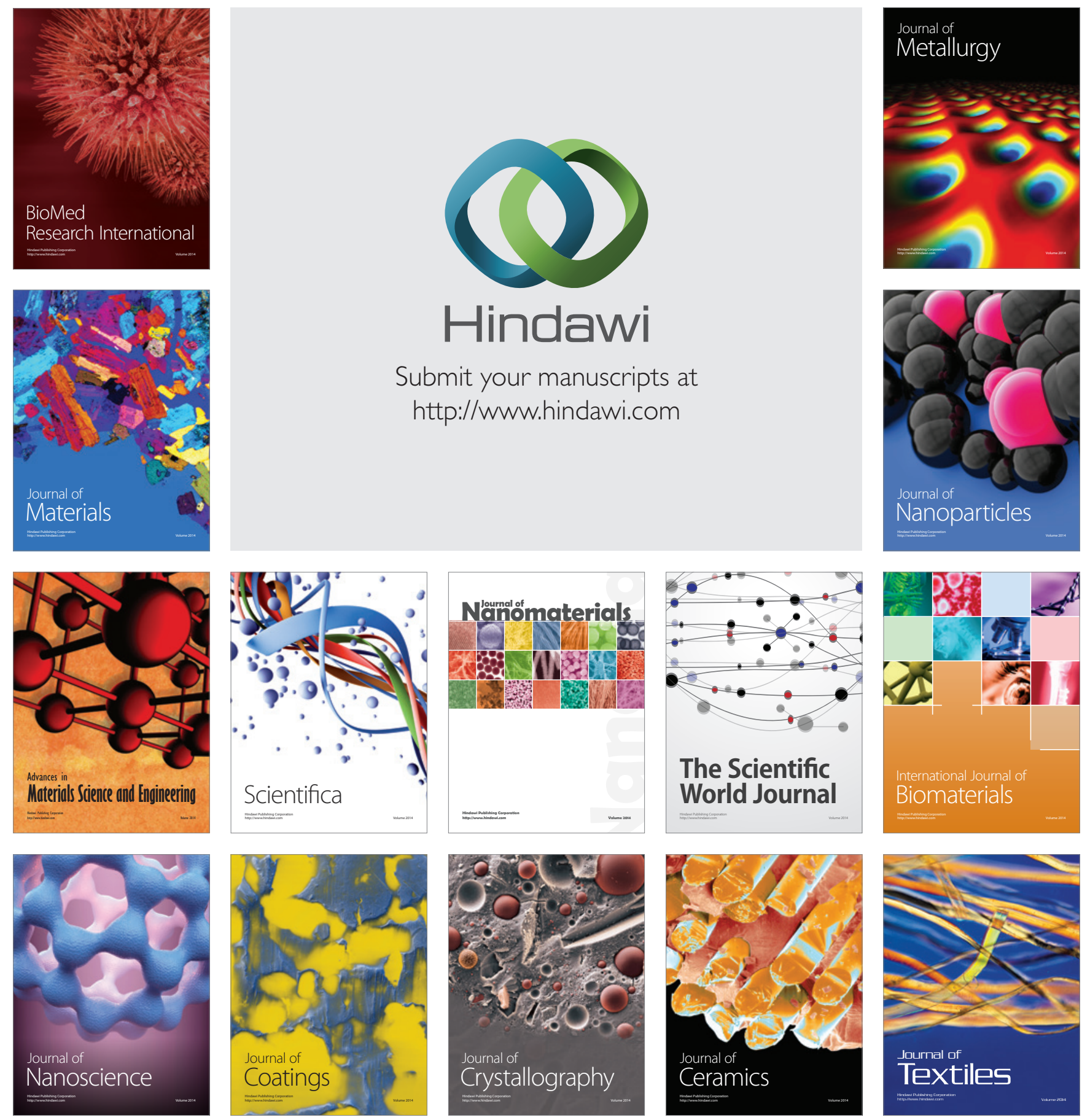